\title{
Pericardial Effusion with Tamponade, an Immune-Related Adverse Event Associated with Nivolumab Therapy: A Case Report and Review of the Literature
}

\author{
Fauzia F. Naime ${ }^{1,2}$ \\ ${ }^{1}$ Leforte Oncologia, São Paulo, Brazil \\ ${ }^{2}$ Instituto do Câncer Dr Arnaldo Vieira de Carvalho, São Paulo, Brazil \\ Email: fauzia.naime@gmail.com
}

How to cite this paper: Naime, F.F. (2018) Pericardial Effusion with Tamponade, an Immune-Related Adverse Event Associated with Nivolumab Therapy: A Case Report and Review of the Literature. Journal of Cancer Therapy, 9, 1039-1047.

https://doi.org/10.4236/jct.2018.912085

Received: December 2, 2018

Accepted: December 26, 2018

Published: December 29, 2018

Copyright $\odot 2018$ by author and Scientific Research Publishing Inc. This work is licensed under the Creative Commons Attribution International License (CC BY 4.0).

http://creativecommons.org/licenses/by/4.0/

\begin{abstract}
Immune checkpoint inhibitors have shown to be very effective when treating lung cancer. Nivolumab, a programmed death-1 (PD-1) inhibitor has been approved for the treatment of metastatic non-squamous and squamous NSCLC. Immunotherapy with checkpoint inhibitors are associated with toxicities defined as immune-related adverse events (irAEs). The most common irAEs are rash, colitis, hepatitis, pneumonitis, and endocrinopathies. Although any organ system can be affected, other rare irAEs can occur and affect the bone marrow, pancreas, and the neurologic and cardiac systems. Pericardial effusion induced by immunotherapy is uncommon and has been described only in a few case reports. Immune-related adverse events usually develop within the first weeks to months after treatment initiation. This is a case report of a 52-year-old male patient with metastatic squamous-cell lung cancer who had a massive pericardial effusion secondary to treatment with nivolumab as second line treatment. During his hospital stay, the patient underwent pericardiocentesis and treatment with corticosteroids. A CT scan after these treatments revealed complete tumor response and minimal pericardial effusion. Nivolumab was definitively ceased. The patient continues to show complete response for 16 months. In general, Nivolumab is well-tolerated and has an excellent safety profile. However, it is important to be aware of life-threatening immune-related adverse events, particularly cardiac toxicity. Consensus guidelines regarding the treatment of the most common irAEs have been established. The optimal management of irAEs is based on clinical experience. It's crucial to report new or rare irAEs to conduct prospective trials to evaluate the best treatment strategy.
\end{abstract}




\section{Keywords}

Pericardial Effusion, Immunotherapy, Nivolumab, Non-Small Cell Lung

Cancer (NSCLC), Immune Related Adverse Events

\section{Introduction}

Nivolumab is a fully human IgG4-programmed death 1 (PD-1) inhibitor belonging to a family of drugs known as immune checkpoint inhibitors associated with toxicities defined as immune-related adverse events (irAEs). Immunotherapy with Nivolumab has shown to be effective in the treatment of non-small cell lung cancer (NSCLC).

Immune-related adverse events commonly affect the gastrointestinal tract, skin, endocrine glands, and the liver and less often the central nervous, cardiovascular, pulmonary, musculoskeletal, and hematologic systems [1]. Although the pathophysiology of irAEs is not yet fully known, it is believed to be related to the role that immune checkpoints play in maintaining immunologic homeostasis [2].

The toxicity profile of checkpoint inhibitors was first described in the studies on ipilimumab, an anti-CTLA-4 antibody used in the treatment of melanoma [3]. Cardiac irAEs are rare, occurring in $<1 \%$ of patients treated with immune checkpoint inhibitors therapy [4].

Pericardial effusion has not been often described as a side effect of nivolumab therapy, being the incidence rate with this drug of 0.06 [5].

This is a case report of a 52-year-old male patient with metastatic squamous-cell lung cancer who had a massive pericardial effusion secondary to treatment with nivolumab as second line treatment.

\section{Case Report}

In February 2016, a 52-year-old man with a history of smoking was diagnosed with squamous-cell carcinoma (PD-L1 inconclusive) with lung and brain metastases (stage IVB). The patient had no previous history of cardiovascular disease and no previous history of hypertension or rheumatologic disease. CT scan findings showed a 4.7 by $4.0 \mathrm{~cm}$ spiculated mass involving and narrowing the basal bronchi and occluding the bronchus of the upper segment and extending to the lingula. It also showed hilar lymph nodes of the upper left, subcarinal, and paratracheal and lymph nodes of the lower right and left, and subaortic (2.2 by $1.8 \mathrm{~cm})$. Two nodes in the upper lingular segment without pleural or pericardial effusion (Figure 1). An MRI the of brain from March 2016 showed multifocal nodular lesions in the following lobes: 1) left frontal, 2) temporal and right parietal, and 3) right occipital and left upper frontal. The patient then underwent a series of cranial irradiation of the central nervous system at $40 \mathrm{~Gy}, 2$ Gy per fraction, which he completed in May 2016 with complete response. He was monitored clinically and by imaging every 3 months. 


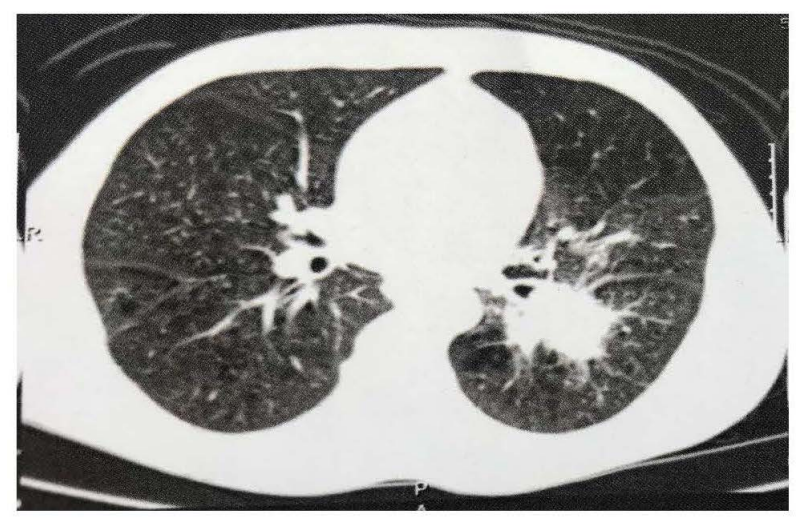

Figure 1. CT scan from February 2016 findings showed a 4.7 by $4.0 \mathrm{~cm}$ spiculated mass involving and narrowing the basal bronchi and occluding the bronchus of the upper segment and extending to the lingula.

The patient was initially treated with concurrent chemotherapy with cisplatin and gemcitabine for six cycles to which he only responded partially.

Because CT scan from November 2016 revealed disease progression in the lung (Figure 2), treatment with nivolumab $3 \mathrm{mg} / \mathrm{kg}$ every 14 days was reinstated in December 2016. After receiving nivolumab for 6 weeks, the patient began to suffer from shortness of breath with progressive deterioration. The patient was hospitalized but declined further treatment with nivolumab after hospital admission. A CT scan revealed massive pericardial effusion (Figure 3).

An echocardiogram showed a massive pericardial effusion with tamponade (measuring $43 \mathrm{~mm}$ and signs of diastolic collapsing of the right chambers i.e., restriction signs).

Ultra-sensitive troponin $\mathrm{T}$ reached $5.3 \mathrm{ng} / \mathrm{dl}(12.7-24.9 \mathrm{ng} / \mathrm{dl})$, CKMB 2.2 $(<6.22 \mathrm{ng} / \mathrm{ml})$, CPK $61 \mathrm{U} / \mathrm{L}$ ( $38-174 \mathrm{U} / \mathrm{L})$, and CBC were unremarkable. The patient underwent pericardiocentesis and the total amount drained was 2500 $\mathrm{cm}^{3}$ of serosanguinous fluid (Table 1). Cytological examination of the pericardial effusion showed no malignant cells. Pathologic examination was also negative for cancer cells. Investigation failed to demonstrate infection, toxin or drug, uremia or acute myocardial infarction.

The patient's condition improved with the pericardiocentesis. Treatment with corticosteroids was initiated with methylprednisolone $2 \mathrm{mg} / \mathrm{kg} /$ daily intravenously during hospitalization. The patient was discharged home after 12 days. Home treatment with steroids, namely prednisone at $1 \mathrm{mg} / \mathrm{kg} / \mathrm{daily}$, was maintained for one month. Sulfamethoxazole trimethoprim was used prophylactic to prevents opportunistic infections.

In February 2017, a CT scan revealed complete tumor response and minimal pericardial effusion. The pericardial effusion did not recur following corticosteroid treatment. In March 2018, a CT scan showed complete response (Figure 4). The patient presented complete response throughout the following 16 months of follow-up. In July 2018, a PET scan showed disease progression in the lung and treatment with docetaxel plus ramucirumab was initiated. 


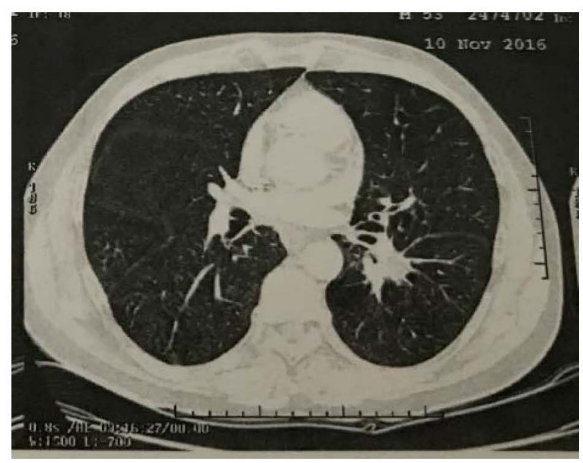

Figure 2. Before Nivolumab: CT scan from november/2016 showed irregular spiculated nodule in the upper segment of the lower lobe of the left lung that surrounds the segmental bronchus, measuring about $3.3 \times 2.4 \mathrm{~cm}$.

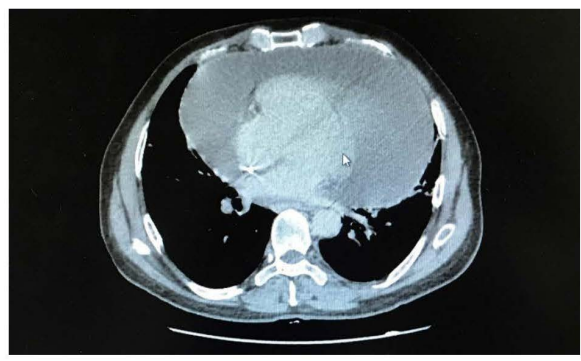

Figure 3. CT scan from February/2017 showed massive pericardial effusion.

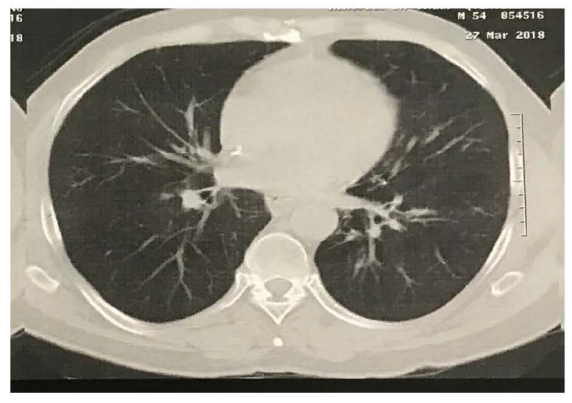

Figure 4. After Nivolumab: Nivolumab was definitively ceased in February/2017 and the CT scan from march/2018 still showed complete response.

Table 1. Pericardiocentesis: Fluid.

\begin{tabular}{cc}
\hline \multicolumn{1}{c}{ Serosanguinous Fluid: } \\
PH & 6.5 \\
WBC & $455 \mathrm{cells} / \mathrm{mm}^{3}$ \\
Neutrophils & $77 \%$ \\
Lymphocytes & $23 \%$ \\
Total protein & $5.7 \mathrm{~g} / \mathrm{dl}$ \\
Albumin & $2.8 \mathrm{~g} / \mathrm{dl}$ \\
Amylase & $43 \mathrm{u} / 1$ \\
LDH & $2234 \mathrm{u} / 1$
\end{tabular}




\section{Discussion}

Nivolumab, which is an anti-programmed death-1 (PD-1) antibody has been approved for the treatment of metastatic non-squamous and squamous-cell carcinoma. It has been demonstrated that it can increase survival [6] [7]. Compared with docetaxel, nivolumab has shown a median overall survival of 9.2 months versus 6.0 months (hazard ratio (HR) $0.59,95 \%$ CI $0.44-0.79$ ) in squamous NSCLC and 12.2 months versus 9.4 months (96\% CI $0.73,0.59-0.89)$ in non-squamous NSCLC, respectively. In both phase 3 trials, pericardial effusion has not been described as an adverse event.

Treatment with immune checkpoint inhibitors is accompanied by immune-related adverse events [2] [8]. The most common irAEs are rash, colitis, hepatitis, pneumonitis, and endocrinopathies. Consensus regarding the treatment of these irAEs has been established [5] [9].

In case reports of myocarditis, $\mathrm{T}$-cell clones infiltrating the myocardium were identical to those present in tumors and skeletal muscle, leading to the speculation that these selective clonal T-cell populations may have reacted against a common, shared antigen of normal myocardium tissue and tumor [10]. Pericarditis and pericardial effusion induced by immunotherapy has been unfrequently described. The first case reports of pericardial effusion was described with ipilimumab in 2015 and 2016 [11] [12]. The pericardial effusion as a side effect of nivolumab was primarily described by Nesfeder et al. (2016), [13]. They reported a case of non-malignant pericardial effusion that progressed, after initiation of nivolumab, to tamponade physiology. Others five cases of nivolumab causing pericardial effusion were reported in the literature [14] [15] [16] [17]. Cardiac tamponade also occurred in a patient with lung cancer in whom tuberculous pericarditis had developed due to a hypersensitivity-related response 3 months after nivolumab treatment was initiated [18]. As far as I know, this is the seventh case of nivolumab-associated pericardial effusion described in the literature and the case with the largest volume drained $\left(2500 \mathrm{~cm}^{3}\right)$.

Immune-related adverse events usually develop within the first weeks to months after treatment initiation but can be present at any time, including after cessation of immune checkpoint blockade therapy [2].

A recent observational, retrospective, pharmacovigilance study (used VigiBase, WHO's global database of individual case safety reports) identified 31.321 cardiovascular adverse events reported in patients who received immune checkpoint inhibitors [19]. Pericardial diseases in patients with lung cancer treated with anti-PD-1 or anti-PD-L1 antibodies were over-reported (49) [56\%] of 87 patients. Cardiovascular irAEs occurred soon after immune checkpoint inhibitors administration with a median time to the onset of pericardial diseases of 30 days (9 - 90).

Some studies suggest a possible correlation between tumor regression and the magnitude of autoimmune toxicity, especially toxicities grades 3 and 4 . Tumor regression was seen in multiple organs and most objective cancer regressions have been long-lasting [20] [21]. A retrospective study based on landmark and 
multivariable analyses in patients with advanced or recurrent NSCLC treated with nivolumab in second-line settings or later revealed that irAEs were associated with nivolumab efficacy [22]. In this study, when setting the landmark at 6 weeks, the median progression free survival with irAES was 9.2 months (95\% CI 4.4 to NR (not reached)) and 4.8 months without irAEs (95\% CI 3.0 to 7.5 ) (p $=0.04)$. Median overall survival were with irAEs NR ( $95 \%$ CI 12.3 to NR) and 11.1 months (95\% CI 9.6 to NR) ( $\mathrm{p}=0.01$ ) without irAEs.

In this case report, our patient presented with pericardial effusion and cardiac tamponade 6 weeks after treatment initiation. Pericardiocentesis was performed and steroid treatment was based on established severe pneumonitis treatment [5]. It is important to plan for a gradual withdrawal of corticoids, whose complete withdrawal should take place in at least 30 days. If corticoids are stopped in less than 30 days, the patient may relapse at the same rate as before.

The most commonly used first-line immunosuppressive agent to treat this excessive immunity against normal organs are glucocorticoids followed by additional immunosuppressive agents if glucocorticoids are initially ineffective in more severe cases. The most frequently used immunosuppressive agents are infliximab and, in the case of hepatitis, Mycophenolate mophetil (MMF), followed by Anti-thymocyte globulin (ATG) in steroid-refractory cases [23] [24].

The prophylactic use of sulfamethoxazole trimethoprim prevents opportunistic infections at a steroid equivalent dose of $1 \mathrm{mg} / \mathrm{kg}$ [25].

A more aggressive medical management, often including high doses of corticosteroids, may be adopted to control and even reverse all autoimmune toxicities [20]. Life-threatening toxicity, including cardiac, pulmonary or neurologic toxicities in particular is an absolute contraindication to restarting treatment with immune checkpoint blockage [2]. A retrospective study evaluated the possibility of retreating patients with advanced NSCLC with checkpoint inhibitors after the occurrence of irAEs. The safety and benefit of retreatment remains unknown. They recommend that patients requiring hospitalization for an initial irAEs and those who have already achieved a complete or partial response before an initial irAEs should not be retreated [26].

\section{Conclusions}

Pericardial effusion as side effect of nivolumab is rare. The management of autoimmune cardiac toxicity requires special consideration due to its potentially lethal nature unless aggressively treated. Most of the toxic effects of immune checkpoints inhibitors are reversible if properly treated and death from irAEs is uncommon.

Multidisciplinary team work is needed to early recognition and unifying management strategies of irAEs in order to optimize the safety of anti-PD-1 and anti-PD-L1 therapy.

\section{Consent for Publication}

Written informed consent was obtained from the patient's next of kin for publi- 
cation of this case report and any accompanying images.

\section{Conflicts of Interest}

Not Applicable.

\section{References}

[1] Weber, J.S., Hodi, F.S., Wolchok, J.D., Topalian, S.L., Schadendorf, D., Larkin, J., Sznol, M., Long, G.V., Li, H., Waxman, I.M., et al. (2017) Safety Profile of Nivolumab Monotherapy: A Pooled Analysis of Patients with Advanced Melanoma. Journal of Clinical Oncology, 35, 785-792. https://doi.org/10.1200/JCO.2015.66.1389

[2] Postow, M.A., Sidlow, R. and Hellmann, M.D. (2018) Immune-Related Adverse Events Associated with Immune Checkpoint Blockade. The New England Journal of Medicine, 378, 158-168. https://doi.org/10.1056/NEJMra1703481

[3] Hodi, F.S., O’Day, S.J., McDermott, D.F., Weber, R.W., Sosman, J.A., Haanen, J.B., Gonzalez, R., Robert, C., Schadendorf, D., Hassel, J.C., et al. (2010) Improved Survival with Ipilimumab in Patients with Metastatic Melanoma. The New England Journal of Medicine, 363, 711-723. https://doi.org/10.1056/NEJMoa1003466

[4] Palmieri, D.J. and Carlino, M.S. (2018) Immune Checkpoint Inhibitor Toxicity. Current Oncology Reports, 20, 72. https://doi.org/10.1007/s11912-018-0718-6

[5] Johnson, D.B., Balko, J.M., Compton, M.L., Chalkias, S., Gorham, J., Xu, Y., et al. (2016) Fulminant Myocarditis with Combination Immune Checkpoint Blockade. The New England Journal of Medicine, 375, 1749-1755.

https://doi.org/10.1056/NEJMoa1609214

[6] Borghaei, H., Paz-Ares, L., Horn, L., Spigel, D.R., Steins, M., Ready, N.E., Chow, L.Q., Vokes, E.E., Felip, E., Holgado, E., et al. (2015) Nivolumab versus Docetaxel in Advanced Nonsquamous Non-Small-Cell Lung Cancer. The New England Journal of Medicine, 373, 1627-1639. https://doi.org/10.1056/NEJMoa1507643

[7] Brahmer, J., Reckamp, K.L., Baas, P., Crinò, L., Eberhardt, W.E., Poddubskaya, E., Antonia, S., Pluzanski, A., Vokes, E.E., Holgado, E., et al. (2015) Nivolumab versus Docetaxel in Advanced Squamous-Cell Non-Small-Cell Lung Cancer. The New England Journal of Medicine, 373, 123-135. https://doi.org/10.1056/NEJMoa1504627

[8] Friedman, C.F., Proverbs-Singh, T.A. and Postow, M.A. (2016) Treatment of the Immune-Related Adverse Effects of Immune Checkpoint Inhibitors: A Review. JAMA Oncology, 2, 1346-1353. https://doi.org/10.1001/jamaoncol.2016.1051

[9] Michot, J.M., Bigenwald, C., Champiat, S., Collins, M., Carbonnel, F., Postel-Vinay, S., Berdelou, A., Varga, A., Bahleda, R., Hollebecque, A., Massard, C., et al. (2016) Immune-Related Adverse Events with Immune Checkpoint Blockade: A Comprehensive Review. European Journal of Cancer, 54, 139-148. https://doi.org/10.1016/j.ejca.2015.11.016

[10] Johnson, D.B., Balko, J.M., Compton, M.L., Chalkias, S., Gorham, J., Xu, Y., Hicks, M., Puzanov, I., Alexander, M.R., Bloomer, T.L., et al. (2016) Fulminant Myocarditis with Combination Immune Checkpoint Blockade. The New England Journal of Medicine, 375, 1749-1755. https://doi.org/10.1056/NEJMoa1609214

[11] Yun, S., Vincelette, N.D., Mansour, I., Hariri, D. and Motamed, S. (2015) Late Onset Ipilimumab-Induced Pericarditis and Pericardial Effusion: A Rare but Life Threatening Complication. Case Reports in Oncological Medicine, 2015, 794842.

[12] Dasanu, C.A., Jen, T. and Skulski, R. (2017) Late-Onset Pericardial Tamponade, Bi- 
lateral Pleural Effusions and Recurrent Immune Monoarthritis Induced by Ipilimumab Use for Metastatic Melanoma. Journal of Oncology Pharmacy Practice, 23, 231-234. https://doi.org/10.1177/1078155216635853

[13] Nesfeder, J., Elsensohn, A.N., Thind, M., Lennon, J. and Domsky, S. (2016) Pericardial Effusion with Tamponade Physiology Induced by Nivolumab. International Journal of Cardiology, 222, 613-614. https://doi.org/10.1016/j.ijcard.2016.08.023

[14] Kushnir, I. and Wolf, I. (2017) Nivolumab-Induced Pericardial Tamponade: A Case Report and Discussion. Cardiology, 136, 49-51. https://doi.org/10.1159/000447053

[15] Vittorio, A., Sharma, R., Siejka, D., Bhattarai, K. and Hardikar, A. (2018) Recurrent Pericardial Effusion While Receiving Nivolumab for Metastatic Lung Adenocarcinoma: Case Report and Review of the Literature. Clinical Lung Cancer, 19, e717-e720. https://doi.org/10.1016/j.cllc.2018.05.010

[16] Shaheen, S., Mirshahidi, H., Nagaraj, G. and Hsueh, C.T. (2018) Conservative Management of Nivolumab-Induced Pericardial Effusion: A Case Report and Review of Literature. Experimental Hematology \& Oncology, 7, 11. https://doi.org/10.1186/s40164-018-0104-y

[17] Kolla, B.C. and Patel, M.R. (2016) Recurrent Pleural Effusions and Cardiac Tamponade as Possible Manifestations of Pseudoprogression Associated with Nivolumab Therapy-A Report of Two Cases. Journal for ImmunoTherapy of Cancer, 4, 80.

[18] Chu, Y.C., Fang, K.C., Chen, H.C., Yeh, Y.C., Tseng, C.E., Chou, T.Y. and Lai, C.L. (2017) Pericardial Tamponade Caused by a Hypersensitivity Response to Tuberculosis Reactivation after Anti-PD-1 Treatment in a Patient with Advanced Pulmonary Adenocarcinoma. Journal of Thoracic Oncology, 12, e111-e114.

[19] Salem, J.E., Manouchehri, A., Moey, M., Lebrun-Vignes, B., Bastarache, L., Pariente, A., Gobert, A., Spano, J.P., Balko, J.M., Bonaca, M.P., Roden, D.M., Johnson, D.B. and Moslehi, J.J. (2018) Cardiovascular Toxicities Associated with Immune Checkpoint Inhibitors: An Observational, Retrospective, Pharmacovigilance Study. The Lancet Oncology, 19, 1579-1589. https://doi.org/10.1016/S1470-2045(18)30608-9

[20] Attia, P., Phan, G.Q., Maker, A.V., Robinson, M.R., Quezado, M.M., Yang, J.C., Sherry, R.M., Topalian, S.L., Kammula, U.S., Royal, R.E., et al. (2005) Autoimmunity Correlates with Tumor Regression in Patients with Metastatic Melanoma Treated with Anti-Cytotoxic T-Lymphocyte Antigen-4. Journal of Clinical Oncology, 23, 6043-6053.

[21] Downey, S.G., Klapper, J.A., Smith, F.O., Yang, J.C., Sherry, R.M., Royal, R.E., Kammula, U.S., Hughes, M.S., Allen, T.E., Levy, C.L., et al. (2007) Prognostic Factors Related to Clinical Response in Patients with Metastatic Melanoma Treated by CTL-Associated Antigen-4 Blockade. Clinical Cancer Research, 13, 6681-6688. https://doi.org/10.1158/1078-0432.CCR-07-0187

[22] Haratani, K., Hayashi, H., Chiba, Y., Kudo, K., Yonesaka, K., Kato, R., Kaneda, H., Hasegawa, Y., Tanaka, K., Takeda, M., et al. (2018) Association of Immune-Related Adverse Events with Nivolumab Efficacy in Non-Small-Cell Lung Cancer. JAMA Oncology, 4, 374-378. https://doi.org/10.1001/jamaoncol.2017.2925

[23] Puzanov, I., Diab, A., Abdallah, K., Bingham 3rd, C.O., Brogdon, C., Dadu, R., et al. (2017) Managing Toxicities Associated with Immune Checkpoint Inhibitors: Consensus Recommendations from the Society for Immunotherapy of Cancer (SITC) Toxicity Management Working Group. Journal for ImmunoTherapy of Cancer, 5, 95. https://doi.org/10.1186/s40425-017-0300-Z

[24] Haanen, J.B.A.G., Carbonnel, F., Robert, C., Kerr, K.M., Peters, S., Larkin, J., Jordan, K., ESMO Guidelines Committee (2017) Management of Toxicities from Im- 
munotherapy: ESMO Clinical Practice Guidelines for Diagnosis, Treatment and Follow-Up. Annals of Oncology, 28, iv119-iv142.

[25] Kronbichler, A., Jayne, D.R. and Mayer, G. (2015) Frequency, Risk Factors and Prophylaxis of Infection in ANCA-Associated Vasculitis. European Journal of Clinical Investigation, 45, 346-368. https://doi.org/10.1111/eci.12410

[26] Santini, F.C., Rizvi, H., Plodkowski, A.J., Ni, A., Lacouture, M.E., Gambarin-Gelwan, M., Wilkins, O., Panora, E., Halpenny, D.F., Long, N.M., Kris, M.G., Rudin, C.M., Chaft, J.E. and Hellmann, M.D. (2018) Safety and Efficacy of Re-Treating with Immunotherapy after Immune-Related Adverse Events in Patients with NSCLC. Cancer Immunology Research, 6, 1093-1099.

https://doi.org/10.1158/2326-6066.CIR-17-0755

\section{List of Abbreviations}

irAEs: Immune-Related Adverse Events;

CT: Computed Tomography;

NSCLC: Non-Small Cell Lung Cancer;

PD-1: Programmed Death 1;

CBC: Complete Blood Count;

NR: Not Reached;

MMF: Mycophenolate Mophetil;

ATG: Anti-Thymocyte Globulin. 